\title{
Point-of-Care Ultrasound: Applications in Low- and Middle-Income Countries
}

\author{
Timothy T. $\operatorname{Tran}^{1}$ (D) $\cdot$ Maung Hlaing ${ }^{1} \cdot$ Martin Krause $^{1}$ \\ Accepted: 21 December 2020 / Published online: 6 January 2021 \\ (C) The Author(s), under exclusive licence to Springer Science+Business Media, LLC part of Springer Nature 2021
}

\begin{abstract}
Purpose of Review This review highlights the applications of point-of-care ultrasound in low- and middle-income countries and shows the diversity of ultrasound in the diagnosis and management of patients.

Recent Findings There is a paucity of data on point-of-care ultrasound in anesthesiology in low- and middle-income countries. However, research has shown that point-of-care ultrasound can effectively help manage infectious diseases, as well as abdominal and pulmonary pathologies.

Summary Point-of-care ultrasound is a low-cost imaging modality that can be used for the diagnosis and management of diseases that affect low- and middle-income countries. There is limited data on the use of ultrasound in anesthesiology, which provides clinicians and researchers opportunity to study its use during the perioperative period.
\end{abstract}

Keywords Low- and middle-income countries · Anesthesiology $\cdot$ Point-of-care ultrasound $\cdot$ Regional anesthesia $\cdot$ Trauma Lung $\cdot$ Cardiac $\cdot$ Abdominal $\cdot$ Breast $\cdot$ HIV $\cdot$ Tuberculosis $\cdot$ Tele-medicine

\section{Introduction}

Ultrasound has been used for a variety of reasons to improve patient safety and medical management. In the USA, traditional diagnostic ultrasound image acquisition is often performed by a sonographer and then interpreted by an imaging specialist physician, such as a radiologist or cardiologist. Point-of-care ultrasound (POCUS) differs from the traditional use of surface ultrasound, as it is defined as a focused exam that is performed and interpreted by the operator.

POCUS as a diagnostic tool is most commonly used in critical care and emergency medicine, but its adoption into

$\overline{\text { This article is part of the Topical Collection on Global Health Anesthesia }}$

Timothy T. Tran

ttran@wustl.edu

Maung Hlaing

maung.hlaing@cuanschutz.edu

Martin Krause

martin.krause@cuanschutz.edu

1 University of Colorado - Anschutz Medical Campus, Mail Stop B113, Leprino Building, 12401 E 17th Ave., Aurora, CO 80045, USA general anesthesia practice is becoming more prevalent. Portable POCUS machines are commonly used in hospitals in the USA, but now smaller handheld probes, which can connect to smartphones or tablets, have been developed. With handheld probes as inexpensive as $\$ 2000$, its use has proliferated over the last several years. Furthermore, POCUS can assist with the diagnosis of illnesses and guidance of procedures while minimizing direct patient contact, making it an excellent tool in epicenters of infectious outbreaks where personal protective equipment (PPE) is often scarce [1]. Lastly, portable probes with improved resolution allow for utilization in out-of-hospital settings such as natural catastrophes [2].

This review article seeks to discuss POCUS in anesthesiology and other relevant specialties in low- and middle-income countries (LMICs).

\section{Search Strategy}

Searches were performed using PubMed (US National Library of Medicine, Bethesda, MD) and Google Scholar (Google Inc., Mountain View, CA) using terms such as "Low and Middle Income," "LMIC," "anesthesiology," "ultrasound," "point of care ultrasound," "POCUS," and other 
relevant search phrases. Only English-language articles were included in this review.

\section{Use of Ultrasound in Anesthesiology: Applications and Obstacles}

Surface ultrasound in anesthesiology is often performed for procedural guidance for regional anesthesia or vascular access. However, POCUS can also be used as a diagnostic tool in anesthesiology. Exams looking at gastric volume [3], inferior vena cava size [4], and diaphragmatic movement [5] can be helpful to manage patients in the perioperative period. In LMICs, ultrasound use in anesthesia is limited by several factors, such as the availability of machines and the opportunity to acquire ultrasound skills. A survey of anesthesiologists in Colombia showed that only $62.7 \%$ of respondents had access to an ultrasound machine for central venous catheter placement [6]. An in-depth narrative was published about the barriers of establishing a regional anesthesia service in Rwanda [7]. In addition to a shortage of regional nerve block needles and supplies, there was a lack of adequately trained providers and local stakeholders. Raiten et al. [8] provided insight on the development of ultrasound in Nepal and Bangladesh for transesophageal echocardiography and POCUS. The authors acknowledged the difficulty of providing education, feedback, and quality assurance for novice sonographers.

Despite these barriers, there is published literature about ultrasound utilization in anesthesia provided in LMICs. A study conducted in Uganda compared the use of ultrasoundguided transversus abdominus plane block versus a sham block showing a decrease in pain at 8,16 , and $24 \mathrm{~h}$, respectively [9]. In addition, a study from Egypt demonstrated that an ultrasound-guided technique compared to the traditional landmark-guided technique for spinal anesthetics significantly improved the first-attempt success rate $(80 \%$ vs $38 \%$, $p<0.001)[10]$.

Besides its use in anesthesia, cardiothoracic and abdominal POCUS are often used in other relevant specialties in LMICs such as trauma, infectious disease, gynecology, and telehealth.

\section{Cardiothoracic Ultrasound}

There is a paucity of data describing cardiac ultrasound in LMICs despite the significant burden of cardiovascular disease in these countries, and significantly worse outcomes compared to high-income countries [11]. One possible explanation for the lack of widespread adoption of POCUS may be the limitations in treatment options for significant cardiac diseases. Confirming a diagnosis such as severe congestive heart failure by ultrasound becomes less useful when treatment options are limited.

Instead, cardiac ultrasound utilization in LMICs has focused on infectious diseases prevalent in these regions. Rheumatic heart disease has a significant burden of illness in LMICs, and several studies have shown that rheumatic heart disease can be reliably diagnosed with handheld ultrasound [12-17]. However, other conditions, such as Chagas cardiomyopathy in South America and endomyocardial fibrosis in Africa, have received little attention despite a significant burden of disease. Investigation in the realm of ultrasound-based diagnosis and management warrants further research $[18,19]$.

Pulmonary and pleural POCUS have proven to play an essential role in the Emergency Rooms and Critical Care Units of high-income countries [20, 21]. POCUS does not expose patients to radiation and has also shown to be more sensitive and specific in diagnosing many pulmonary conditions than chest radiography (CXR) [22-24]. It is a fast and low-risk imaging modality to diagnose respiratory conditions such as diffuse pulmonary edema, and focal consolidations like pneumonia, pneumo- or hemothoraces, and pleural effusions [20].

Pathologic interstitial findings such as vertical B-lines or alveolar consolidation (hepatization with air bronchogram), as well as echogenic debris, fluid, or absent sliding between the parietal and visceral pleura, assist diagnosing the above conditions with high accuracy $[21,25]$. Physiologic signs are horizontal A-lines and lung sliding with a typical M-mode pattern referred to as the seashore sign. In patients with acute respiratory failure, the absence of pathologic signs on thoracic ultrasound combined with a thrombosis on venous sonography can further help to diagnose a pulmonary embolism (sensitivity $81 \%$, specificity $99 \%$ ) [25]. A negative venous analysis and A-lines often suggest asthma or COPD exacerbations (sensitivity 89\%, specificity 97\%) [25].

The Bedside Lung Ultrasound in Emergency (BLUE) algorithm was designed to differentiate the cause of acute respiratory failure in a systematic but rapid fashion [25]. In short, each lung is scanned in 6 zones (upper and lower anterior, lateral, and posterolateral zones). First, the examiner detects the presence or absence of lung sliding and then identifies the type of interstitial artifact (A- or B-lines) by scanning both lungs' anterior chest wall. The findings allow classification into an A- (lung sliding and A-lines), $\mathrm{A}^{\prime}$ - (no lung sliding but A-lines), B- (lung sliding and B-lines), B'- (no lung sliding but B-lines), A/B- (mixed A and B profile), or C-profile (lung consolidation). The B-profile is indicative of diffuse pulmonary edema, while the $\mathrm{B}^{\prime}-$, A/B-, and C-profiles are highly suggestive of pneumonia. If a lung point sign (transition between presence and absence of lung sliding) can be identified in a patient with an A'-profile, a pneumothorax is the most likely diagnosis. The A-profile requires further scanning: if a venous analysis identifies signs of a deep venous thrombosis, 
a pulmonary embolism is likely. If the venous analysis is negative, scanning of the posterolateral chest wall might identify pleural effusions in conjunction with lung consolidation. The so-called Posterolateral Alveolar and/or Pleural Syndrome (PLAPS) is highly indicative of pneumonia. An A-profile in the presence of respiratory failure but in the absence of any pathologic findings often suggests asthma or COPD exacerbations.

The Fluid Administration Limited by Lung Sonography (FALLS) protocol is designed to assist with the diagnosis and management of circulatory failure and prevent pulmonary edema [26]. It rules out obstructive and cardiogenic shock and assists with the management of hypovolemic shock. It also detects volume overload with repeated lung ultrasound and a change from A-lines to B-lines. However, the FALLS protocol has not been evaluated in randomized studies.

Chest ultrasound is also helpful in conditions most prevalent in LMICs. Malaria is responsible for 500 million infections and one million deaths each year [27]. A common cause of death in these patients is malaria-associated acute respiratory distress syndrome (ARDS) [28]. ARDS carries a higher mortality rate in middle-income countries compared to highincome countries [29]. The Kigali modifications of the Berlin criteria have helped to diagnose ARDS in resource-scarce circumstances by allowing ultrasound to identify bilateral pulmonary edema instead of CXR or computed tomography [30]. Identification of bilateral B- or C-profile can help diagnose ARDS in patients suffering from sepsis or severe malaria [28].

Other causes of acute respiratory failure, such as GuillainBarre syndrome, are relevant to LMICs due to a high prevalence of triggering diseases such as HIV [31] and Zika virus [32]. Low diaphragm muscle mass detected by ultrasound was associated with higher risks of complications from acute respiratory failure and prolonged intubation [33]. Chest ultrasound may help to detect patients with ARDS or at risk for respiratory complications, who can then be referred to centers that can provide a higher level care.

POCUS has further contributed to the safety of invasive procedures such as thoracentesis and chest tube placement [23]. Furthermore, lung and pleural POCUS is cost-effective and an easily acquired skill, which has made it an ideal imaging modality for LMICs lacking trained radiologists, CXR, and CT $[34,35]$.

\section{Abdominal Ultrasound}

Ultrasound is a tool that can be used for the diagnosis of many abdominal pathologies. Typhoid, for example, is common in Central and South America, the Middle East, Africa, and parts of Asia [36].

An observational study of 350 patients with typhoid fever performed in Iraq showed the sonographic changes that occurred during the treatment phase [37]. Findings such as hepatomegaly, splenomegaly, bowel wall thickening, and prominent intrahepatic bile ducts were found. The author argued that in atypical cases, in which serology testing was negative, ultrasound could be used to confirm the diagnosis if the clinical presentation was suggestive of typhoid.

One of the severe complications of typhoid is bowel perforation due to hyperplasia and necrosis of the ileum. A study performed in Tanzania looked at the prognostic factors for mortality in patients who presented with bowel perforation secondary to typhoid [38]. One of the conclusions was that ultrasound was superior to chest or abdominal $\mathrm{x}$-rays in the detection of free air.

In addition to bowel perforation, bowel obstruction can be diagnosed using ultrasound. A meta-analysis by Gottlieb et al. found that ultrasound was able to detect a small-bowel obstruction (SBO) with a sensitivity of $92.4 \%$ and specificity of $96.6 \%$ compared to CT [39]. The applicability to LMICs is unclear as there are no research studies, to our knowledge, which systematically evaluate SBO using ultrasound in LMIC. However, there are case reports in which ultrasound was used to diagnose bowel obstruction. A case report published from Uganda demonstrated how abdominal ultrasound was used to diagnose and promptly treat an intussusception in a 5-month-old male [40]. Furthermore, the report showed how emergency care practitioners who are non-physicians trained in emergency care were able to successfully use POCUS.

\section{Infectious Diseases}

Like thoracic ultrasound, abdominal ultrasound in LMICs mostly focuses on point-of-care applications in diagnosing and treating infectious disease.

\section{Malaria}

Studies have incorporated abdominal ultrasound as part of a malaria screening scoring system. Zha et al. evaluated sonography of the liver, spleen, and optic nerve sheath diameter (ONSD) for diagnosing malaria. ONSD has previously been shown to correlate with intracranial hypertension due to cerebral malaria [41]. Splenomegaly and hepatomegaly were very specific for malaria, making ultrasound a useful confirmatory test for malaria [41].

\section{Echinococcosis}

The use of ultrasound for the diagnosis and management of echinococcosis holds promise. Echinococcosis is a parasitic disease caused by tapeworms. This can lead to the development of cysts in the liver and other organs. The cysts can grow and lead to abdominal pain, nausea, and vomiting. If ruptured, 
it can also lead to severe anaphylaxis and death. Echinococcosis is an endemic in the Mediterranean region, at the southern tip of South America, in Central Asia, Siberia, and western China [42].

Given the cystic nature of intra-abdominal infections with Echinococcus, ultrasound is a useful modality to guide needle aspirations of cystic echinococcosis (CE). The World Health Organization statement on CE favors ultrasound over CT and magnetic resonance imaging (MRI) as the imaging of choice for diagnosing and classifying CE [43]. Several studies have examined using a Focused Assessment with Sonography for Echinococcus (FASE) protocol and found easy adoption and use of ultrasound technologies, including handheld ultrasounds, in many resource-limited areas [44]. The FASE protocol involves scanning the abdominal cavity in the transverse, sagittal, oblique subcostal, and coronal supracostal plane [44]. Studies have also shown the ability of FASE to aid treatment regimens or follow response to treatment of leishmaniasis and viral hemorrhagic fever [45] as many of these pathogens lead to cysts or fluid collections, which can easily be examined by ultrasound. Many possibilities for POCUS exist, and further research is certainly warranted.

\section{Human Immunodeficiency Virus and Coinfections}

As many respiratory complications in Sub-Saharan African countries are related to human immunodeficiency virus (HIV)-related infections such as tuberculosis and Pneumocystis jirovecii, ultrasound protocols have been established to identify a combination of findings specific to certain opportunistic infections [46, 47]. Given that bronchoscopes and CT scanners are scarce in LMICs, ultrasound could become crucial for diagnosing other opportunistic pulmonary infections in HIV-positive patients.

The HIV epidemic has led to a resurgence of extrapulmonary tuberculosis (EPTB) in sub-Saharan African countries [48]. EPTB is more frequently seen in patients coinfected with HIV and can manifest in several organs throughout the body [49].

The Fast Assessment with Sonography of HIV/ tuberculosis (FASH) protocol has been used to identify features of EPTB [35]. The FASH protocol consists of nine windows to evaluate for pericardial and pleural effusions, ascites, splenic and liver lesions, and peri-aortic lymph nodes. In locations without access to CT scanners, POCUS becomes the only imaging modality to diagnose EPTB [45]. In addition, the FASH protocol can be taught within 2 days allowing trainees to identify abnormal findings with a sensitivity and specificity of more than $95 \%$ compared to an experienced examiner [35]. One limitation of FASH is the complexity of the protocol, which requires a level of expertise that may be difficult to achieve without appropriate training. Furthermore, reproducibility and reliability of this protocol have not been well studied.

Other opportunistic infections have also been associated with specific lung findings on POCUS. While unilateral pleural effusions are highly suggestive of TB, bilateral effusions are frequently seen in Kaposi's sarcoma [50]. In a retrospective study of HIV-positive patients, asymmetric B-lines, subpleural consolidations, and cystic changes were suggestive of Pneumocystis jirovecii pneumonia (PJP), while consolidations with airbronchogram or pleural effusions were not observed in patients with PJP [47]. These findings correlate with typical CT abnormalities in patients with PJP, in which patchy ground-glass opacities and cystic changes are common, while pulmonary consolidations and pleural effusions often raise the suspicion of an alternate diagnosis [51]. As a bronchoalveolar lavage is oftentimes required to establish the diagnosis of PJP, POCUS could play an important role in settings in which bronchoscopes are not readily available [47]. Furthermore, a case series observed a reduction of B-lines on POCUS correlating with clinical improvement in patients diagnosed with PJP and suggested that POCUS could be a useful tool to confirm treatment response [52].

Last, as HIV remains endemic in Sub-Saharan African countries, patients with HIV will continue to present for surgery, either secondary to HIV-related complications or for elective surgical interventions [53]. A shortage of personal protective equipment (PPE) may put health care workers at risk for contracting HIV while caring for these patients in the perioperative setting [54]. POCUS can help anesthesiologists and surgeons to identify patients with HIV-related complications and guide procedures while minimizing direct patient contact.

\section{Ultrasound in Trauma}

Trauma causes more death in LMICs than malaria, HIV, and TB combined [55]. The Focused Assessment with Sonography of Trauma (FAST) protocol assesses the pericardial and intraperitoneal space for free fluid [56]. The extended FAST (eFAST) protocol includes the FAST protocol's standard views and adds coronal views of the supradiaphragmatic pleural space with the high-depth curvilinear probe and sagittal views of the anterior pleural space with a high-resolution linear probe. The extended protocol can detect a hemothorax, pneumothorax, and pleural effusions with higher sensitivity and specificity than CXR $[21,57,58]$.

Although ultrasound has become an essential part of trauma care in many high-income countries, the adoption of this technology remains limited in LMICs. This underutilization of FAST may be a representation of the lack of resources for surgical-based care in LMICs in comparison to resources for infectious disease pathologies [55]. Only a few limited case series from LMICs investigated the use of POCUS in trauma settings. For example, Nnamonu et al. examined the use of 
trauma ultrasound in a city in Nigeria [59] and found that abdominal ultrasound had high sensitivity for free fluid but poor test characteristics for parenchymal injury.

\section{Breast Ultrasound}

Breast ultrasound is a non-invasive affordable technique that can help differentiate if a palpable mass is concerning for malignancy, an abscess, cyst, and other pathologies. In 2010 , the estimated number of deaths due to breast cancer was 425,000 , with over $50 \%$ occurring in LMICs [60]. Based on the 2010-2016 data from the National Cancer Institute, the 5-year breast cancer survival in the USA ranges from 28 to $98 \%$ compared to $40-60 \%$ in LMICs [61].

The clinical breast exam (CBE) is no longer recommended for screening by the United States Preventative Services Task Force nor the American Cancer Society [62, 63], and imaging is now recommended. In high-income countries, mammography and MRI are used for the detection of breast cancer. In LMICs, these imaging modalities would likely be cost-prohibitive. Sood et al. conducted a meta-analysis on the use of ultrasound to detect breast cancer in LMICs and found a pooled diagnostic sensitivity and specificity of $89 \%$ and 99\% [64]. The study concluded that ultrasound could be used in LMIC to detect breast cancer. A different meta-analysis was completed evaluating the effectiveness of ultrasound in detecting breast cancer following a positive CBE [65]. The authors found a median sensitivity and specificity of $84 \%$ and $94 \%$ in LMIC compared to $94 \%$ and $87 \%$ in high-income countries. The authors determined that ultrasound could triage which patients would need a biopsy after a positive CBE.

In order to better enhance the detection capabilities of ultrasound, Love et al. looked at using computer-aided software to help detect breast cancer [66]. Novice sonographers learned how to perform a breast ultrasound in patients with palpable masses. These images were then analyzed by a software, which was able to detect the difference between benign and malignant masses.

Lastly, POCUS can help with the management of breast abscesses. Chandika et al. showed that ultrasound-guided needle aspiration was more cost-effective and showed no difference in healing rates compared to open incision and drainage [67].

\section{Tele-ultrasound in Low- and Middle-Income Countries}

Another area of interest is the use of tele-medicine to expand ultrasound capabilities in LMICs. With the advent and popularity of cost-effective, portable, and fully functional ultrasound probes, the availability of ultrasound equipment is becoming less a restrictive barrier to POCUS. However, adequate training and longitudinal availability of qualified sonographers for quality assurance continue to be a problem. Therefore, tele-medicine may play a critical role by creating access to expertise for learners while limiting travel and time commitment of experts. Nascimento et al. [16] demonstrated that tele-ultrasound screening for rheumatic and structural heart disease was effective in identifying a significant number of abnormalities in rural Brazil. Sekar et al. [68] showed that tele-ultrasound could be performed with good image quality and allowed diagnoses of cardiac defects in children in Aragonda, India. Moreover, Bhavnani et al. [17] was able to show improvement in outcomes, specifically decreased time to referral, and decreased risk of hospitalization and death when tele-ultrasound technologies were used in a structural heart disease screening clinic in rural India.

Although limited in numbers, these studies show the many possibilities of remote teaching and interpretation for cardiac ultrasound in many LMICs. In fact, Britton et al. conducted a literature review showing that remote ultrasound can be used for both teaching and clinical use to improve patient outcomes in applications outside of cardiac ultrasound, including abdominal and obstetric ultrasound [69]. With the significant time and monetary cost of international travel, teleultrasound remains an intriguing option to build up the POCUS skill sets of local providers in LMICs.

\section{Limitations of Ultrasound in Low- and Middle-Income Countries}

While there are significant benefits to the application of POCUS, a lack of continuous education remains a barrier to its utilization in LMICs [70]. Henwood et al. [71] published a guide on how to develop sustainable POCUS educational programs in resource-limited countries. Amongst others, the guide discusses considerations such as the conduction of a needs assessment, development of a local curriculum, machine management, and quality assurance. Furthermore, supply constraints, such as ultrasound gel and probe covers, limit ultrasound use in LMICs [7]. Low-cost locally funded supplies could present a more sustainable solution than infrequent and expensive external donations.

Last, non-standardized documentation of ultrasound reports can cause miscommunication for patient care. Using accepted reporting systems, such as the American College of Radiology BI-RADS terminology for breast ultrasound, could improve transition of information and care [72].

\section{Conclusion}

The clinical application of ultrasound in LMIC in anesthesia remains limited, but opportunities exist for education and 
research. Other specialties have utilized POCUS to optimize the diagnosis and management of disease states. The relatively low cost of ultrasound compared to other imaging modalities may lend itself to more implementation for procedural guidance and, more importantly, as a diagnostic tool. The low barrier to entry provides an opportunity to perform more research examining the role of POCUS in limited resource settings and LMICs.

\section{Compliance with Ethical Standards}

Conflict of Interest None of the authors has any potential conflicts of interest to disclose.

\section{References}

1. Henwood PC. Imaging an outbreak - ultrasound in an Ebola treatment unit. N Engl J Med. 2019;381(1):6-9.

2. Shah $\mathrm{S}$, et al. Impact of portable ultrasound in trauma care after the Haitian earthquake of 2010. Am J Emerg Med. 2010;28(8):970-1.

3. Perlas, A., et al., Ultrasound assessment of gastric content and volume. Anesthesiology, 2009. 111(1): p. 82-89.

4. Zhang J, Critchley LA. Inferior vena cava ultrasonography before general anesthesia can predict hypotension after induction. Anesthesiology. 2016;124(3):580-9.

5. Tsui JJ, Tsui BC. A novel systematic ABC approach to diaphragmatic evaluation (ABCDE). Can J Anaesth. 2016;63(5):636-7.

6. Calvache JA, et al. Ultrasound guidance for central venous catheterisation. A Colombian national survey. Int J Qual Health Care. 2018;30(8):649-53.

7. Ho $\mathrm{M}$, et al. Barriers and facilitators to implementing a regional anesthesia service in a low-income country: a qualitative study. Pan Afr Med J. 2019;32:152.

8. Raiten J, et al. Perioperative point-of-care ultrasound and transesophageal echocardiography in resource- limited settings-a focus on Nepal and Bangladesh. J Cardiothorac Vasc Anesth. 2020;34(10):2604-10.

9. Kagwa, S., et al., Ultrasound guided transversus abdominis plane versus sham blocks after caesarean section in an Ugandan village hospital: a prospective, randomised, double-blinded, single-centre study. Lancet, 2015. 385 Suppl 2: p. S36.

10. Abdelhamid SA, Mansour MA. Ultrasound-guided intrathecal anesthesia: does scanning help? Egyptian Journal of Anaesthesia. 2019;29(4):389-94.

11. Roth GA, et al. Global, regional, and national burden of cardiovascular diseases for 10 causes, 1990 to 2015. J Am Coll Cardiol. 2017;70(1):1-25.

12. Beaton A, et al. The utility of handheld echocardiography for early diagnosis of rheumatic heart disease. J Am Soc Echocardiogr. 2014;27(1):42-9.

13. Ploutz M, et al. Handheld echocardiographic screening for rheumatic heart disease by non-experts. Heart. 2016;102(1):35-9.

14. Mirabel, M., et al., Screening for rheumatic heart disease: evaluation of a focused cardiac ultrasound approach. Circ Cardiovasc Imaging, 2015. 8(1).

15. Colquhoun SM, et al. Pilot study of nurse-led rheumatic heart disease echocardiography screening in Fiji-a novel approach in a resource-poor setting. Cardiol Young. 2013;23(4):546-52.

16. Nascimento, B.R., et al., Comparison between different strategies of rheumatic heart disease echocardiographic screening in Brazil: data from the PROVAR (Rheumatic Valve Disease Screening Program) Study. J Am Heart Assoc, 2018. 7(4).

17. Bhavnani SP, et al. A randomized trial of pocket-echocardiography integrated mobile health device assessments in modern structural heart disease clinics. JACC Cardiovasc Imaging. 2018;11(4):54657.

18. Mocumbi AO, et al. Endomyocardial fibrosis: an update after 70 years. Curr Cardiol Rep. 2019;21(11):148.

19. Vieira JL, et al. Chagas cardiomyopathy in Latin America review. Curr Cardiol Rep. 2019;21(2):8.

20. Emergency Ultrasound Imaging Criteria Compendium. Ann Emerg Med, 2016. 68(1): p. e11-48.

21. Raheja R, et al. Application of lung ultrasound in critical care setting: a review. Cureus. 2019;11(7):e5233.

22. Irwin Z, Cook JO. Advances in point-of-care thoracic ultrasound. Emerg Med Clin North Am. 2016;34(1):151-7.

23. Brogi E, et al. Thoracic ultrasound for pleural effusion in the intensive care unit: a narrative review from diagnosis to treatment. Crit Care. 2017;21(1):325.

24. Amatya Y, et al. Diagnostic use of lung ultrasound compared to chest radiograph for suspected pneumonia in a resource-limited setting. Int J Emerg Med. 2018;11(1):8.

25. Lichtenstein DA, Meziere GA. Relevance of lung ultrasound in the diagnosis of acute respiratory failure: the BLUE protocol. Chest. 2008;134(1):117-25.

26. Lichtenstein DA. BLUE-protocol and FALLS-protocol: two applications of lung ultrasound in the critically ill. Chest. $2015 ; 147(6)$ : 1659-70.

27. Mohan A, Sharma SK, Bollineni S. Acute lung injury and acute respiratory distress syndrome in malaria. J Vector Borne Dis. 2008;45(3):179-93.

28. Leopold SJ, et al. Point-of-care lung ultrasound for the detection of pulmonary manifestations of malaria and sepsis: an observational study. PLoS One. 2018;13(12):e0204832.

29. Laffey JG, et al. Geo-economic variations in epidemiology, patterns of care, and outcomes in patients with acute respiratory distress syndrome: insights from the LUNG SAFE prospective cohort study. Lancet Respir Med. 2017;5(8):627-38.

30. Riviello ED, et al. Hospital incidence and outcomes of the acute respiratory distress syndrome using the Kigali modification of the Berlin definition. Am J Respir Crit Care Med. 2016;193(1):52-9.

31. Thornton CA, Latif AS, Emmanuel JC. Guillain-Barre syndrome associated with human immunodeficiency virus infection in Zimbabwe. Neurology. 1991;41(6):812-5.

32. Cao-Lormeau VM, et al. Guillain-Barre syndrome outbreak associated with Zika virus infection in French Polynesia: a case-control study. Lancet. 2016;387(10027):1531-9.

33. Sklar MC, et al. Association of low baseline diaphragm muscle mass with prolonged mechanical ventilation and mortality among critically ill adults. JAMA Netw Open. 2020;3(2):e1921520.

34. Brogi $\mathrm{E}$, et al. Could the use of bedside lung ultrasound reduce the number of chest $\mathrm{x}$-rays in the intensive care unit? Cardiovasc Ultrasound. 2017;15(1):23.

35. Heller T, et al. Short course for focused assessment with sonography for human immunodeficiency virus/tuberculosis: preliminary results in a rural setting in South Africa with high prevalence of human immunodeficiency virus and tuberculosis. Am J Trop Med Hyg. 2010;82(3):512-5.

36. Centers for Disease Control and Prevention. Typhoid fever. [cited 202030 September]; Available from: https://wwwnc.cdc.gov/ travel/diseases/typhoid.

37. Younis SN. The role of abdominal ultrasound in the diagnosis of typhoid fever: an observational study. Travel Med Infect Dis. 2014;12(2):179-82.

38. Chalya, P.L., et al., Typhoid intestinal perforations at a University teaching hospital in Northwestern Tanzania: a surgical experience 
of 104 cases in a resource-limited setting. World J Emerg Surg, 2012. 7: p. 4.

39. Gottlieb M, et al. Utilization of ultrasound for the evaluation of small bowel obstruction: a systematic review and meta-analysis. Am J Emerg Med. 2018;36(2):234-42.

40. Stolz LA, et al. Intussusception detected with ultrasound in a resource-limited setting. Lancet. 2013;381(9882):2054.

41. Murphy S, et al. Ultrasound findings in Plasmodium falciparum malaria: a pilot study. Pediatr Crit Care Med. 2011;12(2):e58-63.

42. World Health Organization. Echinococcosis: epidemiology \& risk factors. [cited 202022 September]; Available from: https://www. who.int/echinococcosis/epidemiology/en/.

43. Eckert, J., et al., WHO/OIE manual on echinococcosis in humans and animals : a public health problem of global concern / edited by J. Eckert ... [et al.]. 2001, Paris, France : World Organisation for Animal Health.

44. Del Carpio M, et al. Early diagnosis, treatment and follow-up of cystic echinococcosis in remote rural areas in Patagonia: impact of ultrasound training of non-specialists. PLoS Negl Trop Dis. 2012;6(1):e1444.

45. Belard S, et al. Point-of-care ultrasound assessment of tropical infectious diseases-a review of applications and perspectives. Am J Trop Med Hyg. 2016;94(1):8-21.

46. Ndege, R., et al., Sonography to rule out tuberculosis in SubSaharan Africa: a prospective observational study. Open Forum Infect Dis, 2019. 6(4): p. ofz154.

47. Giordani MT, et al. Point-of-care lung ultrasound for diagnosis of Pneumocystis jirovecii pneumonia: notes from the field. Crit Ultrasound J. 2018;10(1):8.

48. Shivakoti R, et al. Association of HIV infection with extrapulmonary tuberculosis: a systematic review. Infection. 2017;45(1):11-21.

49. Golden MP, Vikram HR. Extrapulmonary tuberculosis: an overview. Am Fam Physician. 2005;72(9):1761-8.

50. Heller T, et al. Focused assessment with sonography for HIVassociated tuberculosis (FASH): a short protocol and a pictorial review. Crit Ultrasound J. 2012;4(1):21.

51. Orlowski HLP, et al. Imaging spectrum of invasive fungal and fungal-like infections. Radiographics. 2017;37(4):1119-34.

52. Limonta $\mathrm{S}$, et al. Lung ultrasound in the management of pneumocystis pneumonia: a case series. Int J STD AIDS. 2019;30(2):188-93.

53. Rickard J, et al. Surgical infections in low- and middle-income countries: a global assessment of the burden and management needs. Surg Infect. 2020;21(6):478-94.

54. Gupta S, Wong EG, Kushner AL. Scarcity of protective items against HIV and other bloodborne infections in 13 low- and middle-income countries. Tropical Med Int Health. 2014;19(11): 1384-90.

55. Gosselin, R.A., et al., Injuries: the neglected burden in developing countries. Bull World Health Organ, 2009. 87(4): p. 246-246a.
56. Ollerton JE, et al. Prospective study to evaluate the influence of FAST on trauma patient management. J Trauma. 2006;60(4):78591.

57. Brooks A, et al. Emergency ultrasound in the acute assessment of haemothorax. Emerg Med J. 2004;21(1):44-6.

58. Kirkpatrick AW, et al. Hand-held thoracic sonography for detecting post-traumatic pneumothoraces: the Extended Focused Assessment with Sonography for Trauma (EFAST). J Trauma. 2004;57(2):288 95.

59. Nnamonu MI, et al. Diagnostic value of abdominal ultrasonography in patients with blunt abdominal trauma. Niger J Surg. 2013;19(2): 73-8.

60. Anderson BO, Ilbawi AM, El Saghir NS. Breast cancer in low and middle income countries (LMICs): a shifting tide in global health. Breast J. 2015;21(1):111-8.

61. Rivera-Franco MM, Leon-Rodriguez E. Delays in breast cancer detection and treatment in developing countries. Breast Cancer (Auckl). 2018;12:1178223417752677.

62. Oeffinger $\mathrm{KC}$, et al. Breast cancer screening for women at average risk: 2015 guideline update from the American Cancer Society. JAMA. 2015;314(15):1599-614.

63. Siu, A.L. and U.S.P.S.T. Force, Screening for breast cancer: U.S. Preventive Services Task Force Recommendation Statement. Ann Intern Med, 2016. 164(4): p. 279-296.

64. Sood R, et al. Ultrasound for breast Cancer detection globally: a systematic review and meta-analysis. J Glob Oncol. 2019;5:1-17.

65. Tsu, V., et al., Breast ultrasound following a positive clinical breast examination: does it have a role in low-and middle-income countries? Journal of Global Radiology, 2015. 1(2).

66. Love SM, et al. Palpable breast lump triage by minimally trained operators in Mexico using computer-assisted diagnosis and lowcost ultrasound. J Glob Oncol. 2018;4:1-9.

67. Chandika $\mathrm{AB}$, et al. Ultrasound guided needle aspiration versus surgical drainage in the management of breast abscesses: a Ugandan experience. BMC Res Notes. 2012;5:12.

68. Sekar P, Vilvanathan V. Telecardiology: effective means of delivering cardiac care to rural children. Asian Cardiovasc Thorac Ann. 2007;15(4):320-3.

69. Britton N, et al. Tele-ultrasound in resource-limited settings: a systematic review. Front Public Health. 2019;7:244.

70. Wanjiku GW, Bell G, Wachira B. Assessing a novel point-of-care ultrasound training program for rural healthcare providers in Kenya. BMC Health Serv Res. 2018;18(1):607.

71. Henwood PC, et al. A practical guide to self-sustaining point-ofcare ultrasound education programs in resource-limited settings. Ann Emerg Med. 2014;64(3):277-85 e2.

72. Scheel JR, et al. ACR BI-RADS use in low-income countries: an analysis of diagnostic breast ultrasound practice in Uganda. J Am Coll Radiol. 2016;13(2):163-9.

Publisher's Note Springer Nature remains neutral with regard to jurisdictional claims in published maps and institutional affiliations. 